\title{
Correlation of Narrow Band Imaging with Magnifying Colonoscopy and Histology in Colorectal Tumors
}

\author{
Hee Yong Yoo', Moon Sung Lee', Bong Min Ko¹, Hee Kyung Kim², Hyung Su Ahn', \\ Seung Hyo Han', Jun Yong Bae¹, Seul Ki Min¹, Jong Chan Lee ${ }^{1}$ and Chang Beom Ryu ${ }^{1}$ \\ Departments of ${ }^{1}$ Internal Medicine and ${ }^{2}$ Pathology, Soonchunhyang University Bucheon Hospital, Soonchunhyang University College of Medicine, \\ Bucheon, Korea
}

Background/Aims: Narrow band imaging (NBI) is a new technique that uses optical filters for imaging of mucosal morphology. The aim of this study was to correlate findings of NBI with magnifying colonoscopy and histology for prediction of neoplastic colorectal lesion.

Methods: Between September 2005 and December 2007, 107 colon polyps from 68 patients were detected by conventional colonoscopy and subsequently evaluated by NBI with magnifying colonoscopy and analyzed for a pit pattern and a capillary pattern. More analysis was done regarding thickness and irregularity of capillary features.

Results: Pit pattern with NBI magnification to discriminate between neoplastic and non-neoplastic lesions had a sensitivity of $88.9 \%$ and a specificity of $87.5 \%$; capillary pattern yielded test performance characteristics of $91.9 \%$ and $87.5 \%$. In respect of capillary thickness, invisible capillaries were found significantly more often in hyperplastic lesions. All thick capillaries were found in neoplastic polyps, and found significantly more often in carcinomas with submucosal massive invasion $(\mathrm{sm}-\mathrm{m})(p<0.01)$. In respect of capillary irregularity, invisible capillaries were found significantly more often in hyperplasic lesions, and severely irregular capillaries were found significantly more often in sm-m lesions $(p<0.01)$.

Conclusions: Observation of capillary thickness and irregularity by NBI magnification is useful for correlating histological grade with carcinoma, especially with depth of submucosal invasion.

Key Words: Narrow band imaging; Magnifying colonoscopy; Histology; Colorectal tumor

\section{INTRODUCTION}

Narrow band imaging (NBI) endoscopy is a newly developed technique that uses 2 optical filters to pass only the short (blue/green) wavelengths enhancing the visualization of microvessels and their fine structures on the mucosal surface, based on the fact that the depth of light infiltration depends on its wavelength. The contrast on the vessel areas could be increased by artificially narrowing the wavelength

\footnotetext{
Received: June 21, 2010 Revised: August 28, 2011

Accepted: September 6, 2011

Correspondence: Moon Sung Lee

Department of Internal Medicine, Soonchunhyang University Bucheon Hospital, Soonchunhyang University College of Medicine, 1174 Jung-dong, Wonmi-gu, Bucheon 420-767, Korea

Tel: +82-32-621-5114, Fax: +82-32-621-5662, E-mail: mslee@schmc.ac.kr

(c) This is an Open Access article distributed under the terms of the Creative Commons Attribution Non-Commercial License (http://creativecommons.org/ licenses/by-nc/3.0) which permits unrestricted non-commercial use, distribution, and reproduction in any medium, provided the original work is properly cited.
}

area using filters because the sharpness may be improved by eliminating the wavelength areas with dispersed lights. NBI uses $415 \mathrm{~nm}$ and $540 \mathrm{~nm}$ of wavelength; the former enhances microvessels in the mucosal surface layer with brown image, whereas the latter enhances the submucosal layer or microvessels in the submucosal layer with green image, both enabling detailed observation of mucosal structure and capillary pattern..$^{1-3} \mathrm{NBI}$ is not only useful for the detection of tumors but also for the differential diagnosis between tubular adenoma and hyperplastic polyp. ${ }^{4,5}$

NBI with magnifying endoscopy is reported to be useful for patients with Barrett's esophagus or gastric tumors ${ }^{6,7}$ but was not sufficiently investigated for colorectal disease in South Korea.

There are also very few reports on correlation between NBI and histologic findings. This study observed the structure of mucosal surface and blood vessel and correlated with histologic diagnosis of colorectal tumors. 


\section{MATERIALS AND METHODS}

\section{Patients and colonoscopic observations}

This study was performed in 107 colorectal lesions of 68 patients who received endoscopic resection of colorectal tumors at Soonchunhyang University Hospital in Bucheon, South Korea, between September 2005 and December 2007. These lesions were first detected by conventional view, and then observed by NBI system. Every lesion was detected using white-light colonoscopy (CF-Q240ZI, CF-H260AZI; Olympus, Tokyo, Japan), and EVIS LUCERA system (Olympus Optical, Tokyo, Japan) was used for videoendoscope system. Endoscopic specialists with more than 5 years of experience in the field of colonscopy performed the endoscopic observations in this study. After detailed observation by NBI magnification, all lesions were resected endoscopically. Specimens were pinned to a board and fixed in $10 \%$ buffered formalin for 24 hours. Then these specimens were cut into 2 to 3-mm blocks and performed on hematoxylin and eosinstained sections. Pathologic examination was performed based on the World Health Organization criteria by a single pathologist unaware of the features of each case. Following histologic diagnosis, a total of 107 lesions were categorized into 8 groups based on their histologic grade and depth of submucosal invasion: hyperplastic polyp, inflammatory polyp, low grade tubular adenoma, high grade tubular adenoma, tubulovillous adenoma, villous adenoma, carcinoma with intramucosal-scant submucosal invasion (m-sml), and carcinoma with submucosal massive invasion (sm-m). Submucosal massive invasion was defined as the invasion of more than $1,000 \mu \mathrm{m}$ for this study. ${ }^{8}$

\section{Capillary patterns and features according to NBI magnification (Table 1)}

Pit patterns and capillary patterns were categorized using NBI magnification system (Fig. 1), the former following the Kudo's classification and the latter Sano's classification. Pit pattern types III, IV, and V based on Kudo's classification and capillary pattern types II and III based on Sano's classification were determined as neoplastic lesions., ${ }^{9,10}$

NBI magnification enables the observation of capillary patterns on the surface layer of tumors. Each lesion was further categorized according to thickness and irregularity of their capillary by close observation with NBI magnification. Capillaries were divided into invisible, thin, or thick capillary based on their thickness. Based on NBI findings, lesions without microvessel or of extreme opacity were determined as invisible capillary; lesions with thin pits and even blood vessel diameters throughout the lesion were determined as thin capillary; and mixture of blood vessels small but larger than 'thin' capillaries are determined as thick capillary (Fig. 2). Capillaries were also divided into invisible, regular, or irregular type based on their irregularity. Invisible capillary type was determined by absence of or extremely opaque capillary; regular capillary type by smooth capillary running with apparent regular meshwork of capillaries across the pits and lesion; and irregular capillary type by irregular running of capillaries with apparent irregular meshwork of capillaries across the pits and lesion (Fig. 3). Lesions with thin and thick, or regular and irregular capillaries were determined as neoplastic. These capillary types by NBI and histologic findings were correlated afterwards.

SPSS for windows version 13.0 (SPSS Inc., Chicago, IL, USA) was used for statistical analysis with $\chi^{2}$ test for data analysis. $p$-values of less than 0.05 were determined statistically significant.

\section{RESULTS}

\section{Patients characteristics}

A total of 68 patients ( 49 male, 19 female) were enrolled in the study, and their mean age was 59 years old (range, 42 to 84) (Table 2). White-light colonoscopy and NBI with magnifying colonoscopy were performed in every patient, with successful intubation of cecum. Bowel preparation was well conducted in every test, and lesions in every patient were

Table 1. Capillary Patterns and Capillary Features Identified by NBI Magnification

\begin{tabular}{|c|c|c|}
\hline \multirow{3}{*}{$\begin{array}{l}\text { Capillary } \\
\text { pattern }^{10}\end{array}$} & I & Absent meshed brown capillary vessel \\
\hline & II & Present meshed brown capillary vessel, slightly thicker and loose capillary density \\
\hline & III & Present meshed brown capillary vessel, thicker, branching, irregularity capillary and dense capillary density \\
\hline \multirow{3}{*}{$\begin{array}{l}\text { Capillary } \\
\text { thickness }\end{array}$} & Invisible & \\
\hline & Thin & Thin and even thickness \\
\hline & Thick & Uneven thickness \\
\hline \multirow{3}{*}{$\begin{array}{l}\text { Capillary } \\
\text { irregularity }\end{array}$} & Invisible & \\
\hline & Regular & Capillaries running smoothly between pits and an apparent regular meshwork of capillaries throughout \\
\hline & Irregular & Capillaries running irregularly between pits and an apparent irregular meshwork of capillaries throughout \\
\hline
\end{tabular}

NBI, narrow band imaging. 

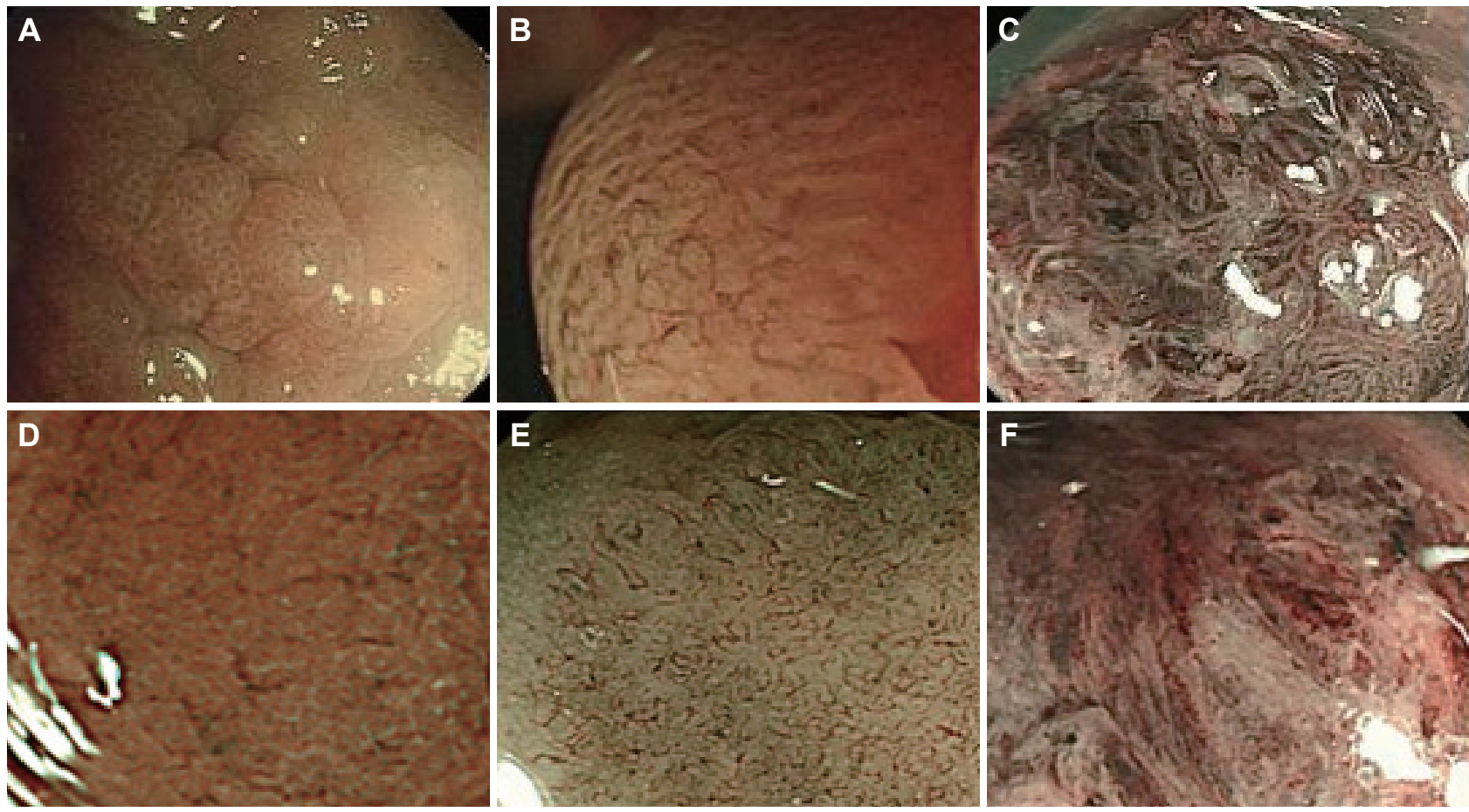

Fig. 1. Classification of capillary pattern. (A, B) Capillary pattern type I shows absence of meshed brown capillary vessel. (C, D) Capillary pattern type II shows presence of meshed brown capillary vessel, slightly thicker and loose capillary density. (E, F) Capillary pattern type III shows presence of meshed brown capillary vessel, thicker, branching, irregularity of capillary and dense capillary density.
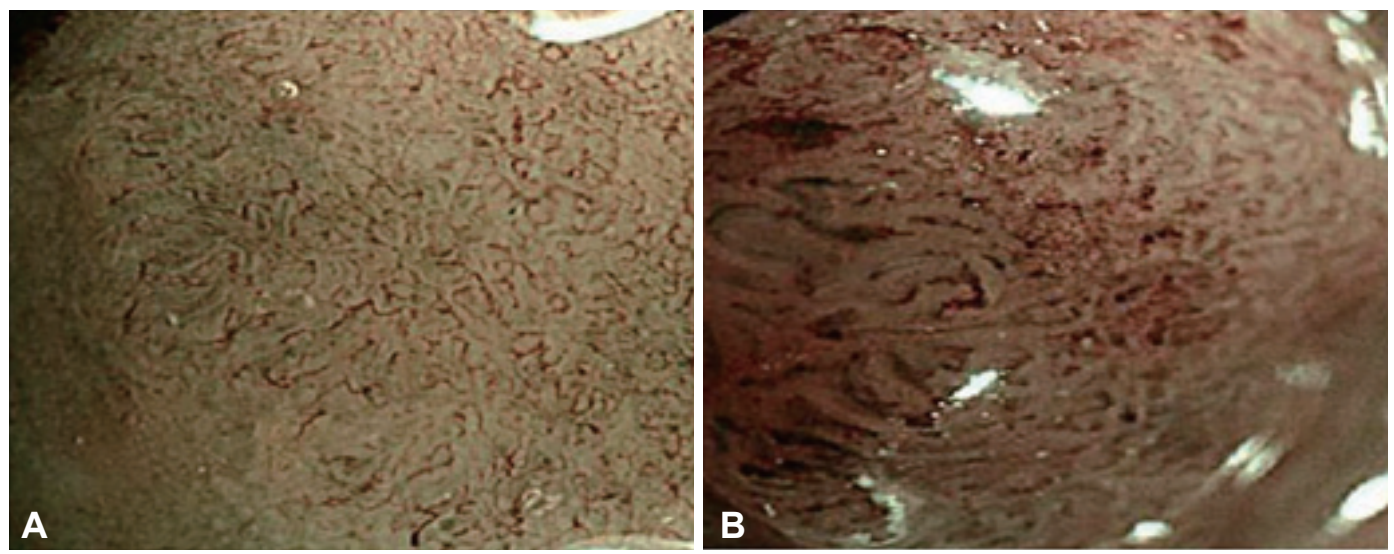

Fig. 2. Classification of microvessel thickness as determined by narrow band imaging. (A) Microvessels were classified as thin when they were thin and even thickness. (B) Microvessels were classified as thick when they were uneven thickness.

categorized proficiently following the observation of colorectal mucosa by NBI.

\section{Lesions and colonoscopic observations}

A total of 107 colon tumors detected by white-light colonoscopy were distributed as follows: ascending colon 18 cases, transverse colon 26 cases, descending colon 14 cases, sigmoid colon 26 cases, and rectum 23 cases. The types of resected tumors were sessile polyp 32 cases (29.9\%), subpedunculated polyp 35 cases $(32.7 \%)$, flat-elevated polyp 18 cases $(16.8 \%)$, laterally spreading tumor 13 cases $(12.1 \%)$, and pedunculated polyp 9 cases $(8.4 \%)$. The mean tumor size was $11.25 \mathrm{~mm}$ ranging from $2 \mathrm{~mm}$ to $40 \mathrm{~mm}$ (Table 2).

\section{Histologic findings of lesions}

Histologic findings of total 107 lesions were non-neoplastic in 8 cases, which were further divided again as 7 cases of hyperplastic polyp and 1 case of inflammatory polyp; the remaining 99 cases were neoplastic, which were divided again as low grade tubular adenoma in 55 cases, high grade tubular adenoma in 16 cases, tubulovillous adenoma in 10 cases, villous adenoma in 2 cases, carcinoma with intramucosal to scant submucosal invasion in 10 cases, and carcinoma with submucosal massive invasion in 6 cases (Table 2). 

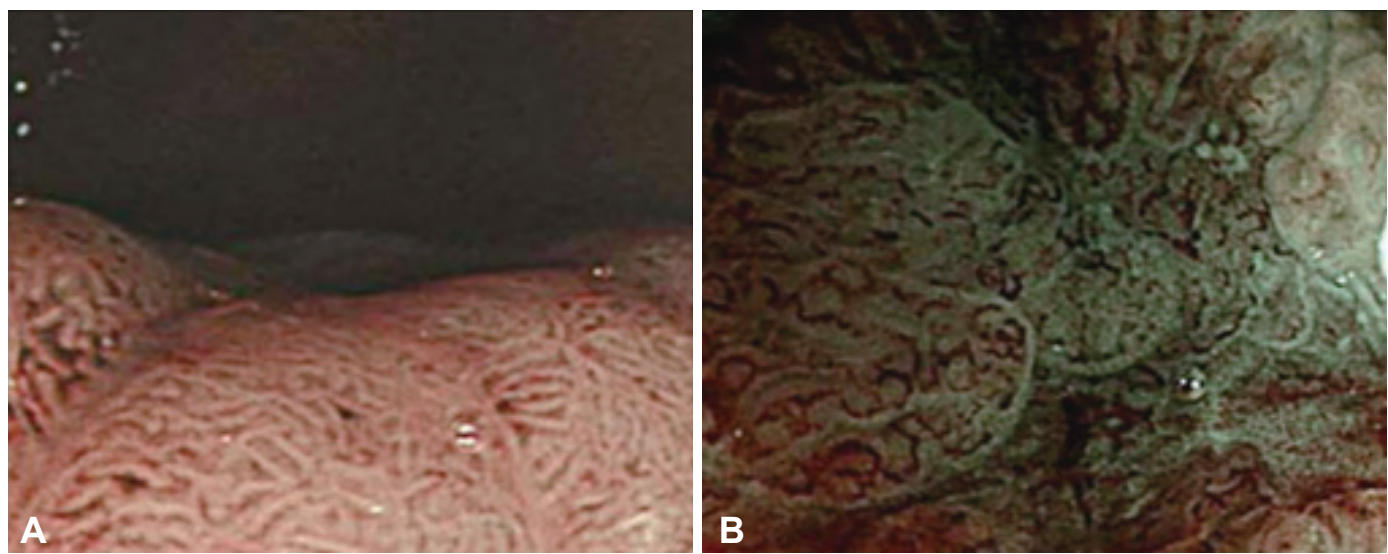

Fig. 3. Classification of microvascular features as determined by narrow band imaging. (A) Microvessels were classified regular when lesions had microvessels running smoothly between pits and an apparent regular meshwork of microvessels throughout. (B) Microvessels were classified irregular when lesions had microvessels running irregularly between pits and an apparent irregular meshwork of microvessels throughout.

Table 2. Demographics of Study Participants and Clinicopathological Characteristics of Study Lesions

\begin{tabular}{|c|c|}
\hline Variable & Value \\
\hline Patients & 68 \\
\hline Sex, M:F & 49:19 \\
\hline Mean age (minimum, maximum) & $59(42,84)$ \\
\hline Lesion & 107 \\
\hline \multicolumn{2}{|l|}{ Location } \\
\hline Ascending colon & 18 \\
\hline Transverse colon & 26 \\
\hline Descending colon & 14 \\
\hline Sigmoid colon & 26 \\
\hline Rectum & 23 \\
\hline \multicolumn{2}{|l|}{ Morphology } \\
\hline Sessile polyp (Is), No. (\%) & $32(29.9)$ \\
\hline Subpedunculated polyp (Isp), No. (\%) & $35(32.7)$ \\
\hline Flat-elevated polyp (IIa), No. (\%) & $18(16.8)$ \\
\hline Pedunculated polyp (Ip), No. (\%) & $9(8.4)$ \\
\hline Laterally spreading tumor (LST) & $13(12.1)$ \\
\hline Mean size in mm (range) & $11.25(2-40)$ \\
\hline \multicolumn{2}{|l|}{ Histolopathology } \\
\hline Non-neoplastic & 8 \\
\hline Hyperplastic polyp & 7 \\
\hline Inflammatory polyp & 1 \\
\hline Neoplastic & 99 \\
\hline Tubular adenoma-low grade & 55 \\
\hline Tubular adenoma-high grade & 16 \\
\hline Tubulovillous adenoma & 10 \\
\hline Villous adenoma & 2 \\
\hline Adenocarcinoma-m-sm1 & 10 \\
\hline Adenocarcinoma-sm-m & 6 \\
\hline
\end{tabular}

$\overline{\mathrm{m} \text {-sm1, intramucosal-scant submucosal invasion; sm-m, submu- }}$ cosal massive invasion.

\section{NBI magnification and histological finding}

Differential diagnosis of neoplastic lesions by Kudo's pit patterns classification on NBI with magnifying colonoscopy showed sensitivity and specificity of $88.9 \%$ and $87.5 \%$, respectively; the positive predictive value and negative predictive value were $98.9 \%$ and $38.9 \%$, respectively. The capillary patterns by Sano's classification also showed high sensitivity and specificity of $91.9 \%$ and $87.5 \%$, respectively; the positive predictive value and negative predictive value were estimated as $98.9 \%$ and $46.7 \%$, respectively. The classification by capillary thickness showed sensitivity and specificity of $99.0 \%$ and $62.5 \%$, respectively; the positive predictive value and negative predictive value were estimated as $97.0 \%$ and $83.3 \%$. The classification by capillary irregularity revealed sensitivity and specificity of $100 \%$ and $62.5 \%$, respectively; the positive predictive value and negative predictive value were estimated as 97.1\% and $100 \%$ (Table 3).

Table 4 shows the relationship between capillary pattern as determined by NBI magnification and histologic findings. Hyperplastic polyp was most common among cases of capillary pattern type I (6/15), while carcinoma accounted $50 \%$ of capillary pattern type III cases (12/24) including submucosal massive invasion of 6 cases, suggesting the increasing trend of carcinoma and submucosal massive invasion toward the capillary pattern type III $(p<0.01)$.

Table 5 shows relationship between capillary thickness as determined by NBI magnification and histologic findings. Invisible capillary was more common among hyperplastic polyp (5/6), while thin capillary was more common among low grade tubular adenoma (35/58). All cases with thick capillary were diagnosed as neoplastic lesions (43/43); 13 cases of them were carcinoma (13/43). Six cases with submucosal massive invasion were all determined as thick capillary $(p<0.01)$. 
Table 3. Diagnostic Accuracy of NBI Colonoscopy

\begin{tabular}{|c|c|c|c|c|c|c|c|c|}
\hline & \multicolumn{2}{|c|}{ Pit pattern } & \multicolumn{2}{|c|}{ Capillary pattern } & \multicolumn{2}{|c|}{ Capillary thickness } & \multicolumn{2}{|c|}{ Capillary irregularity } \\
\hline & Neoplastic & $\begin{array}{c}\text { Non- } \\
\text { neoplastic }\end{array}$ & Neoplastic & $\begin{array}{c}\text { Non- } \\
\text { neoplastic }\end{array}$ & Neoplastic & $\begin{array}{c}\text { Non- } \\
\text { neoplastic }\end{array}$ & Neoplastic & $\begin{array}{c}\text { Non- } \\
\text { neoplastic }\end{array}$ \\
\hline \multicolumn{9}{|l|}{ Histologic diagnosis } \\
\hline Neoplastic & 88 & 11 & 91 & 8 & 98 & 1 & 99 & 0 \\
\hline Non-neoplastic & 1 & 7 & 1 & 7 & 3 & 5 & 3 & 5 \\
\hline Sensitivity, \% & \multicolumn{2}{|c|}{88.9} & \multicolumn{2}{|c|}{91.9} & \multicolumn{2}{|c|}{99.0} & \multicolumn{2}{|c|}{100} \\
\hline Specificity, \% & \multicolumn{2}{|c|}{87.5} & \multicolumn{2}{|c|}{87.5} & \multicolumn{2}{|c|}{62.5} & \multicolumn{2}{|c|}{62.5} \\
\hline PPV, \% & \multicolumn{2}{|c|}{98.9} & \multicolumn{2}{|c|}{98.9} & \multicolumn{2}{|c|}{97.0} & \multicolumn{2}{|c|}{97.1} \\
\hline NPV, \% & \multicolumn{2}{|c|}{38.9} & \multicolumn{2}{|c|}{46.7} & \multicolumn{2}{|c|}{83.3} & \multicolumn{2}{|c|}{100} \\
\hline
\end{tabular}

NBI, narrow band imaging; PPV, positive predictive value; NPV, negative predictive value.

Table 4. Relationship between Capillary Pattern ${ }^{10}$ as Determined by NBI Findings and Histologic Findings

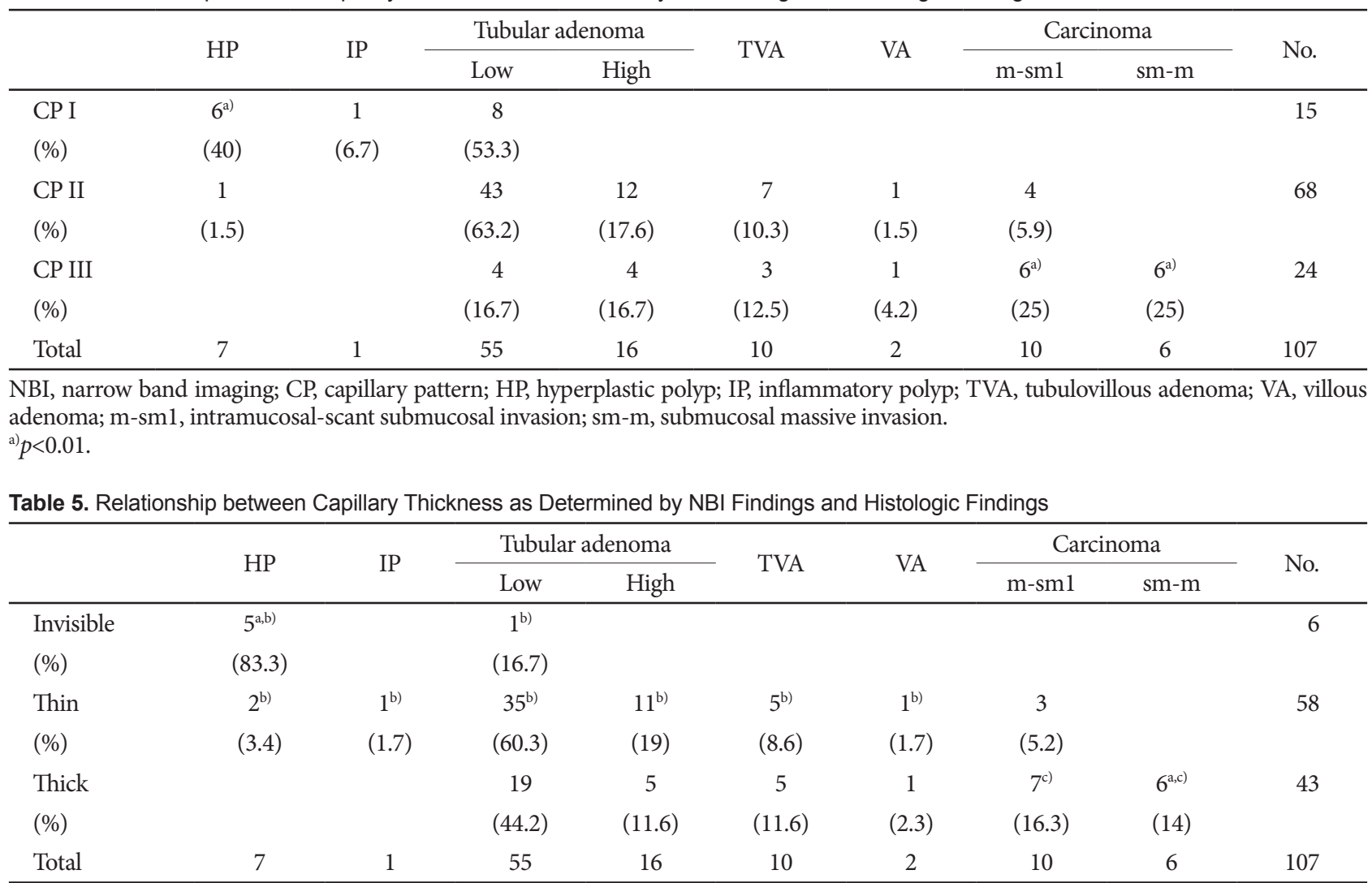

NBI, narrow band imaging; HP, hyperplastic polyp; IP, inflammatory polyp; TVA, tubulovillous adenoma; VA, villous adenoma; m-sm1, intramucosal-scant submucosal invasion; sm-m, submucosal massive invasion.

${ }^{\text {a) }} p<0.01 ;{ }^{\text {b) }}$ vs. ${ }^{\text {c) }} p<0.01$.

Table 6 shows relationship between capillary irregularity as determined by NBI magnification and histologic findings. All hyperplastic polyp cases were determined as invisible capillary (5/5). Regular capillary was more common among low grade tubular adenoma cases (51/78); while irregular capillary was more common among carcinoma cases (12/24) and accounted the whole cases of neoplastic lesions (24/24) and submucosal massive invasion $(6 / 6)(p<0.01)$.

\section{DISCUSSION}

Magnifying endoscopy provides details of the surface of the gastrointestinal tract, and enables pit pattern examination of colorectal tumors by using indigo carmine or cresyl violet., ${ }^{911-14}$ The pit pattern of colorectal tumors (type I-V pit pattern) was suggested by Kudo et al., ${ }^{9,13}$ using stereomicroscopy, and is useful for histologic evaluation of tumors. The pit pattern was developed for differential diagnosis between 
Table 6. Relationship between Capillary Irregularity as Determined by NBI Findings and Histologic Findings

\begin{tabular}{|c|c|c|c|c|c|c|c|c|c|}
\hline & \multirow{2}{*}{$\mathrm{HP}$} & \multirow{2}{*}{ IP } & \multicolumn{2}{|c|}{ Tubular adenoma } & \multirow{2}{*}{ TVA } & \multirow{2}{*}{ VA } & \multicolumn{2}{|c|}{ Carcinoma } & \multirow{2}{*}{ No. } \\
\hline & & & Low & High & & & m-sml & sm-m & \\
\hline Invisible & $5^{\mathrm{a}, \mathrm{b})}$ & & & & & & & & 5 \\
\hline$(\%)$ & $(100)$ & & & & & & & & \\
\hline Regular & $2^{\mathrm{b})}$ & $1^{\mathrm{b})}$ & $51^{\text {b) }}$ & $11^{\mathrm{b})}$ & $8^{\mathrm{b})}$ & $1^{\mathrm{b})}$ & 4 & & 78 \\
\hline (\%) & (2.6) & $(1.3)$ & $(65.4)$ & $(14.1)$ & $(10.3)$ & (1.3) & (5.1) & & \\
\hline Irregular & & & 4 & 5 & 2 & 1 & $6^{c)}$ & $6^{\mathrm{a}, \mathrm{c}}$ & 24 \\
\hline (\%) & & & $(16.7)$ & $(20.8)$ & (8.3) & $(4.2)$ & $(25)$ & $(25)$ & \\
\hline Total & 7 & 1 & 55 & 16 & 10 & 2 & 10 & 6 & 107 \\
\hline
\end{tabular}

NBI, narrow band imaging; HP, hyperplastic polyp; IP, inflammatory polyp; TVA, tubulovillous adenoma; VA, villous adenoma; m-sm1, intramucosal-scant submucosal invasion; sm-m, submucosal massive invasion.

${ }^{\text {a) }} p<0.01$; ${ }^{\text {b) }}$ vs. ${ }^{\text {c) }} p<0.01$.

neoplasia and non-neoplasia, and is used for determining the degree of histologic atypia and depth of early carcinoma, presence of minute residual tumor after endoscopic resection, ${ }^{11-15}$ the degree of histologic inflammation when ulcerative colitis is present, and diagnosis of dysplasia and colitisassociated carcinoma. ${ }^{16-18}$

NBI with magnifying endoscopy is capable of observing both the mucosal pit pattern and surface layer capillary pattern without dye spray but only with mode conversion. ${ }^{3}$ This is why the NBI endoscopy is also called as an electronic chromoendoscopy'. NBI with magnifying endoscopy can indirectly measure capillary pit patterns similar to the regular pit pattern type II, III, and IV, although the capillary pit pattern type $\mathrm{V}$, a marker of carcinoma, is not measured sufficiently yet. ${ }^{5,19-21}$ Capillary evaluation is a useful alternative to the pit patterns for the diagnosis of a colorectal tumor appearing as a type $\mathrm{V}$ pit pattern. Recently, a number of studies are attempting to evaluate capillary features (vessel diameter, irregularity and capillary structure) for the diagnosis of colorectal tumors using NBI magnification., ${ }^{5,1921-25}$

This study classified lesions firstly by the capillary patterns by Sano's classification, and additionally classified by capillary features; that is, thin or thick according to the capillary thickness and regular or irregular according to the capillary irregularity. Thin and regular capillary could be included in the capillary pattern type II, while thick and irregular capillary could be included in the capillary pattern type III, with similar features on tumor observation. Capillary pattern type I was quite common including invisible or faintly visible mucosal capillary meshwork. The capillary meshwork in this type was invisible in general or regional in some cases, and previous biopsy site performed in some cases at other hospitals revealed regional capillary meshwork (Fig. 1B).

The sensitivity and specificity of differential diagnosis between neoplasia or non-neoplasia based on the capillary meshwork of Sano's classification were $96.4 \%$ and $92.3 \%$, re- spectively in a previous study, ${ }^{26}$ and were slightly lower in this study with $91.9 \%$ and $87.5 \%$, respectively. Classification by capillary thickness or irregularity showed higher sensitivity but slightly lower specificity compared to the classification by capillary pattern (Table 3 ).

Among capillary patterns based on the NBI with magnifying endoscopy, neoplastic lesion was slightly more common among capillary pattern type I, carcinoma was more apparent in type III, and all 6 cases of submucosal massive invasion were type III. Among capillary thickness based on the NBI with magnifying endoscopy, invisible capillary was more common among hyperplastic lesions but only 1 case of neoplastic lesions belonged to it. Most carcinoma and all 6 cases of submucosal massive invasion showed thick pattern. Among capillary irregularity, irregular capillary was histologically worse than regular pattern and most common among carcinoma lesions included all 6 cases of submucosal massive invasion. Since capillary pattern by Sano's classification could be interpreted differently between observer, we simplified to capillary thickness or irregularity for this study. The results showed that capillary thickness or irregularity by NBI endoscopy was useful enough to correlate between submucosal massive invasion and histologic finding of colorectal tumor.

This study confirmed that NBI with magnifying endoscopy enables the differential diagnosis between neoplastic and non-neoplastic lesion. ${ }^{1} \mathrm{NBI}$ with magnifying endoscopy was also found useful when determining whether the depth of submucosal invasion is more than $1,000 \mu \mathrm{m}$ or not, a key factor in deciding an endoscopic therapy of early colorectal carcinoma. NBI endoscopy is simple to perform for both endoscopists and patients, and does not require special instrument or dye. The capillary features such as capillary thickness or irregularity by NBI magnification is simpler and easier for clinical use than classifications by pit patterns or capillary patterns. 
NBI with magnifying endoscopy is expected to be widely used clinically for capillary evaluation and proper diagnosis of colorectal tumor. This was a small retrospective study, and the classifications presented in the study require further investigation in multicenter, randomized, controlled trials, regarding the degree of conformity with endoscopic findings and the superiority of NBI colonoscopy compared to conventional colonoscopy in detection and prediction of histologic diagnosis of the tumor.

\section{Conflicts of Interest}

The authors have no financial conflicts of interest.

\section{REFERENCES}

1. Sano Y, Muto M, Tajiri H, Ohtsu A, Yoshida S. Optical/digital chromoendoscopy during colonoscopy using narrow-band imaging system. Dig Endosc 2005;17(Suppl 1):S43-S48.

2. Gono K, Obi T, Yamaguchi M, et al. Appearance of enhanced tissue features in narrow-band endoscopic imaging. J Biomed Opt 2004;9:568-577.

3. Machida H, Sano Y, Hamamoto Y, et al. Narrow-band imaging in the diagnosis of colorectal mucosal lesions: a pilot study. Endoscopy 2004;36:1094-1098.

4. East JE, Suzuki N, Bassett P, et al. Narrow band imaging with magnification for the characterization of small and diminutive colonic polyps: pit pattern and vascular pattern intensity. Endoscopy 2008;40:811-817.

5. Sikka S, Ringold DA, Jonnalagadda S, Banerjee B. Comparison of white light and narrow band high definition images in predicting colon polyp histology, using standard colonoscopes without optical magnification. Endoscopy 2008;40:818-822.

6. Bansal A, Ulusarac O, Mathur S, Sharma P. Correlation between narrow band imaging and nonneoplastic gastric pathology: a pilot feasibility trial. Gastrointest Endosc 2008;67:210-216.

7. Yao K, Iwashita A, Tanabe H, et al. White opaque substance within superficial elevated gastric neoplasia as visualized by magnification endoscopy with narrow-band imaging: a new optical sign for differentiating between adenoma and carcinoma. Gastrointest Endosc 2008;68:574-580.

8. Kitajima K, Fujimori T, Fujii S, et al. Correlations between lymph node metastasis and depth of submucosal invasion in submucosal invasive colorectal carcinoma: a Japanese collaborative study. J Gastroenterol 2004;39:534-543.

9. Kudo S, Tamura S, Nakajima T, Yamano H, Kusaka H, Watanabe H. Diagnosis of colorectal tumorous lesions by magnifying endoscopy. Gastrointest Endosc 1996;44:8-14.

10. Sano Y, Horimatsu T, Fu K, Katagiri A, Muto M, Ishikawa H. Magnifying observation of microvascular architecture of colorectal lesions using a narrow-band system. Dig Endosc 2006;18(Suppl 1):S44-S51.

11. Tanaka S, Haruma K, Nagata S, Oka S, Chayama K. Diagnosis of inva- sion depth in early colorectal carcinoma by pit pattern analysis with magnifying endoscopy. Dig Endosc 2001;13(Suppl 1):S2-S5.

12. Tanaka S, Haruma K, Ito M, et al. Detailed colonoscopy for detecting early superficial carcinoma: recent developments. J Gastroenterol 2000;35 Suppl 12:121-125.

13. Kudo S, Hirota S, Nakajima T, et al. Colorectal tumours and pit pattern. J Clin Pathol 1994;47:880-885.

14. Oka S, Tanaka S, Nagata S, et al. Relationship between histopathological features and type $\mathrm{V}$ pit pattern determined by magnifying video colonoscopy in early colorectal carcinoma. Dig Endosc 2005;17:117122.

15. Nagata S, Tanaka S, Haruma K, et al. Pit pattern diagnosis of early colorectal carcinoma by magnifying colonoscopy: clinical and histological implications. Int J Oncol 2000;16:927-934.

16. Hata K, Watanabe T, Motoi T, Nagawa H. Pitfalls of pit pattern diagnosis in ulcerative colitis-associated dysplasia. Gastroenterology 2004;126:374-376.

17. Kiesslich R, Fritsch J, Holtmann M, et al. Methylene blue-aided chromoendoscopy for the detection of intraepithelial neoplasia and colon cancer in ulcerative colitis. Gastroenterology 2003;124:880-888.

18. Larghi A, Lecca PG, Costamagna G. High-resolution narrow band imaging endoscopy. Gut 2008;57:976-986.

19. Inoue T, Murano M, Murano N, et al. Comparative study of conventional colonoscopy and pan-colonic narrow-band imaging system in the detection of neoplastic colonic polyps: a randomized, controlled trial. J Gastroenterol 2008;43:45-50.

20. Tanaka S, Oka S, Hirata M, Yoshida S, Kaneko I, Chayama K. Pit pattern diagnosis for colorectal neoplasia using narrow band imaging magnification. Dig Endosc 2006;18(Suppl 1):S52-S56.

21. Su MY, Hsu CM, Ho YP, Chen PC, Lin CJ, Chiu CT. Comparative study of conventional colonoscopy, chromoendoscopy, and narrowband imaging systems in differential diagnosis of neoplastic and nonneoplastic colonic polyps. Am J Gastroenterol 2006;101:2711-2716.

22. Nakayoshi T, Tajiri H, Matsuda K, Kaise M, Ikegami M, Sasaki H. Magnifying endoscopy combined with narrow band imaging system for early gastric cancer: correlation of vascular pattern with histopathology (including video). Endoscopy 2004;36:1080-1084.

23. Hirata M, Tanaka S, Oka S, et al. Magnifying endoscopy with narrow band imaging for diagnosis of colorectal tumors. Gastrointest Endosc 2007;65:988-995.

24. Hirata M, Tanaka S, Oka S, et al. Evaluation of microvessels in colorectal tumors by narrow band imaging magnification. Gastrointest Endosc 2007;66:945-952.

25. Rastogi A, Bansal A, Wani S, et al. Narrow-band imaging colonoscopy: a pilot feasibility study for the detection of polyps and correlation of surface patterns with polyp histologic diagnosis. Gastrointest Endosc 2008;67:280-286.

26. Sano Y, Horimatsu T, Fu KI, et al. Magnified observation of microvascular architecture using narrow band imaging (NBI) for the differential diagnosis between non-neoplastic and neoplastic colorectal lesion: a prospective study. Gastrointest Endosc 2006;63(5 Suppl):AB102. 